Rev Biomed 2004; 15:181-190.

\title{
Toxoplasmosis congénita: una mirada al problema.
}

Revisión

Ivonne Martín-Hernández.

Laboratorio de Errores Innatos del Metabolismo. Centro Nacional de Genética Médica. La Habana, Cuba.

\section{RESUMEN.}

Objetivos. Este trabajo ofrece una revisión teórica y actualizada sobre la toxoplasmosis congénita, dirigida no sólo al personal médico especializado en este tema, sino también a otros especialistas interesados en el estudio de esta enfermedad.

Fuentes de extracción. Artículos actualizados y libros de texto relacionados con el tema. Consulta en Internet.

Resultados. El parásito Toxoplasma gondii (T. gondii) es uno de los responsables de la infección congénita grave en el recién nacido, presentando un gran número de signos y secuelas, llegando incluso a producir la muerte. El riesgo de transmisión congénita y la severidad de la infección dependen del período de gestación en que la embarazada se infecte. Mundialmente la incidencia varía de un lugar a otro entre $1: 1000$ y $1: 10000$ de nacidos vivos, dependiendo de la zona geográfica y el nivel socioeconómico de la población. Es de gran importancia el diagnóstico pre y postnatal mediante la búsqueda serológica de anticuerpos específicos o técnicas de detección directa del parásito en aras de la terapeútica temprana que evite daños graves para el feto.

Conclusiones. Dada la importancia que tiene para la salud de la población y en especial de las embarazadas, se pretende llamar la atención sobre la necesidad de retomar un pesquisaje masivo a partir de un examen parasitológico, a nivel primario de salud, en el proceso de captación del embarazo con el propósito de detectar la infección primaria y realizar el seguimiento. (Rev Biomed 2004; 15:181-190)

Palabras clave: Toxoplasmosis congénita, Toxoplasma gondii, embarazada, recién nacidos.

\section{SUMMARY.}

Congenital toxoplasmosis: a look at the problem. Objectives. This work offers a theoretical and upto-date review of congenital toxoplasmosis, directed not only to medical specialists on this topic, but also to other specialists interested in the study of this disease.

Sources of information. Updated articles and books related to the theme. Internet search.

Results. The parasite Toxoplasma gondii (T. gondii)

Solicitud de sobretiros: Lic. Ivonne Martín-Hernández. Centro Nacional de Genética Médica, Centro colaborador de la OMS para el desarrollo de enfoques genéticos en la promoción de salud. Calle 146 No. 3102 esq. ave 31. Marianao C.P. 11600. Ciudad de la Habana. Cuba. Correo electrónico: ivonne.martin@infomed.sld.cu

Recibido el 18/Septiembre/2003. Aceptado para publicación el 21/Enero/2004.

Este artículo está disponible en http://www.uady.mx/sitios/biomedic/revbiomed/pdf/rb041536.pdf

Vol.15/No.3/Julio-Septiembre, 2004 


\section{Martín-Hernández.}

is one of those responsable for serious congenital infection in newborns, presenting a large number of signs and sequels, even causing death. The risk of congenital transmission and the severity of infection depend on the gestation period in which the pregnant woman is infected. Worldwide, incidence varies from one place to another, between 1:1000 and 1:10000 of live newborns, depending on geographical zone and the socioecomomic levels of the population. Pre and postnatal diagnostic is important by means of serologic searching of specific antibodies or by direct detection of the parasite in order to give early therapy and avoid serious damages to the fetus.

Conclusions. Due to the importance it has for the health of the population, and especially for pregnant women, it is our aim to make tre need of reconsidering a massive screening, starting with a parasitologic exam, continuing to the primary level of health, in order to detect the diagnosis of the primary infection in early pregnancy and make a follow up.

\section{(Rev Biomed 2004; 15:181-190)}

Keywords: Congenital toxoplasmosis, Toxoplasma gondii, pregnancy, newborns.

\section{INTRODUCCIÓN.}

La toxoplasmosis es una enfermedad parasitaria producida por el protozoo intracelular obligado Toxoplasma gondii (T. gondii). Está ampliamente extendida por todo el mundo y su frecuencia varía mucho según las zonas geográficas y los hábitos alimentarios. Usualmente la principal vía de transmisión de esta parasitosis es la oral, por ingestión de carnes crudas o semicocidas portadoras de quistes, o por la ingestión de oocistos en el agua o en los alimentos contaminados con las heces de gatos. Son menos frecuentes las vías de transmisión parenteral, respiratorias, mucosal (conjuntival) y cutánea. La transmisión por vía transplacentaria ocurre en las mujeres embarazadas cuando son afectadas por una infección primaria, aunque se han descrito casos raros de infección congénita en infecciones maternas anteriores al embarazo $(1,2)$.

La toxoplasmosis adquirida durante el embarazo es responsable de más defectos congénitos que el herpes, la rubeola y la sífilis juntos y es más común e insidiosa de lo que hasta ahora han creído médicos e investigadores (3). Es por ello de enorme importancia que los especialistas que trabajan en la atención primaria de salud, especialmente aquellos que intervienen en la atención a la mujer embarazada, conozcan los aspectos fundamentales relacionados con las formas de transmisión y prevención de la infección, manifestaciones clínicas, respuesta inmune, interpretación de las pruebas diagnósticas y conducta médica.

En países como Dinamarca, Finlandia, Noruega y Reino Unido $(27,4 \%, 20,3 \%, 10,9 \%$ y 7,7\%, respectivamente donde la seroprevalencia es baja), el porcentaje de mujeres con riesgo de contraer la infección primaria por Toxoplasma gondii durante la gestación es alto, al igual que el riesgo para el feto. A nivel mundial la incidencia de la toxoplasmosis congénita varía de un lugar a otro, pero puede oscilar entre 1:1000 y 1:10000 nacidos vivos dependiendo de la zona geográfica, estilo de vida y nivel socioeconómico de la población (6-13).

Anualmente nacen en Estados Unidos entre 400 y 4000 niños afectados por toxoplasmosis congénita, mientras que en Francia nacen 6 de cada 700 con esta infección $(14,15)$. En cambio, en Suecia donde la incidencia de infección por toxoplasma durante el embarazo es baja, registra una prevalencia de 0.73 por cada 10000 (16). Pesquisajes realizados en Brasil reportan una incidencia de toxoplasmosis congénita al nacer de 8 en 10000 (17).

Los estudios epidemiológicos realizados en Cuba permiten estimar conservadoramente que el porcentaje de positividad a $T$. gondii en gestantes se encuentra entre un 51 y un $75 \%$ y que nacen anualmente alrededor de 250 niños infectados por este parásito, aunque en el momento del nacimiento sólo un pequeño número de ellos presente evidencias clínicas de la infección. Estudios de seroconversión indican tasas de 0.2 a 2.0\%, dependiendo del área de salud estudiada (18-22).

El recién nacido con esta infección presenta un gran número de signos y secuelas, llegándole incluso

\section{Revista Biomédica}




\section{Toxoplasmosis congénita.}

a producir la muerte. Este agente es capaz de atravesar la barrera placentaria y alojarse en los tejidos embrionarios o fetales causando daños de variada magnitud $(3,4)$. Algunos investigadores recomiendan el aborto cuando la infección se presenta antes de las 16 semanas de embarazo. Después de este tiempo, sólo el tratamiento adecuado y el seguimiento ecográfico en busca de alteraciones que sugieran daño fetal serán las guías para determinar la conducta a seguir (4, 23-26).

\section{TRANSMISIÓN TRANSPLACENTARIA.}

Estudios indican que aproximadamente entre el 39 y $50 \%$ de las mujeres infectadas por primera vez durante el embarazo, no tratadas, tienen probabilidad de pasarle la infección al feto. El riesgo y la severidad de la infección al feto dependerán en parte del momento en que se infectó la madre. La mayor severidad se da en los primeros meses del embarazo, teniendo en cuenta que las primeras semanas se consideran las principales etapas del desarrollo intrauterino. El período de tiempo entre la infección de la placenta y la transmisión al feto es variable, entre 4 y 16 semanas, por lo que se debe considerar a la placenta infectada como una fuente potencial de infección al feto durante todo el embarazo $(3,4)$.

Cuando la madre se infecta por primera vez en el último trimestre del embarazo, existe el riesgo de transmisión en el 65\% de los fetos. Esta cifra baja a $25 \%$ y $15 \%$ cuando la infección fue adquirida en el segundo y primer trimestre respectivamente. $\mathrm{La}$ infección en la madre es generalmente benigna o transcurre asintomática. Si la infección fue adquirida antes de la gestación (seis meses o más antes de la concepción), el niño no desarrolla infección congénita. También se plantea que una madre que parió un niño con toxoplasmosis no vuelve a tener otro con la enfermedad. Se han descrito casos de abortos o mortinatos en infecciones recientes, pero no hay evidencia definitiva de abortos a repetición asociados a la toxoplasmosis (2-4).

En las embarazadas inmunosuprimidas por SIDA, linfomas o corticoterapia, la transmisión congénita puede ocurrir, debido a una primoinfección o a una reactivación de la infección por Toxoplasma. En estas pacientes la infección fetal podría ser atenuada o prevenida si hubiese detección precoz de las gestantes seronegativas y un posterior seguimiento serológico de estas; así como por aplicación de terapéutica a las que presentasen serología de infección reciente (2-5).

\section{MANIFESTACIONES CLIINICAS.}

La infección aguda por Toxoplasma en la embarazada, se presenta en un $90 \%$ de los casos en forma asintomática. Los signos clínicos más frecuentes son: adenopatías, fiebre, malestar general, cefalea, mialgias, odinofagia, eritema máculo papular, hepatomegalia y esplenomegalia. Raramente ocurre coriorretinitis, pues es más frecuente en la forma crónica. El leucograma puede mostrar linfocitosis y linfocitos atípicos, lo que obliga a hacer diagnóstico diferencial con infecciones virales como citomegalovirus y mononucleosis infecciosa. En pacientes inmunosuprimidas, puede presentarse compromiso pulmonar o del sistema nervioso central $(1,2)$.

Al nacer, aproximadamente el $75 \%$ de los recién nacidos infectados son asintomáticos y sólo en un $8 \%$ se presenta un severo compromiso del sistema nervioso central u ocular. Los síntomas que aparecen en el recién nacido dependen del momento de la infección del feto y aparecen entre las 3 semanas y los 3 meses de vida del niño e incluso pueden manifestarse años después del nacimiento.

Si la infección ocurre al final del embarazo, se produce una forma aguda generalizada, donde la mitad de los recién nacidos son prematuros y de bajo peso, con un cuadro clínico de tipo séptico caracterizado por fiebre, hepatoesplenomegalia, ictericia y en algunos casos miocarditis o neumonía intersticial. Alrededor del $80 \%$ de ellos tienen líquido cefalorraquídeo (LCR) normal. No se presenta exantema y rara vez existe compromiso neurológico y ocular. La mortalidad de estos niños es elevada y llega al $12 \%$ si no se aplica tratamiento $(1,3,4)$.

Cuando la infección fetal ocurre alrededor de la mitad del embarazo, la etapa de infección generalizada 


\section{Martín-Hernández.}

se produce durante la vida intrauterina y en el momento del nacimiento se encuentra sintomatología de encefalitis. En los casos benignos el niño puede tener peso normal y presentar pocas manifestaciones de la enfermedad, pero después de varias semanas se vuelve apático, con dificultad para comer y ocasionalmente desarrolla convulsiones $(1,3,4)$.

En la toxoplasmosis subclínica la secuela más importante es la retinocoroiditis, encontrándose un $75 \%$ de los casos con lesiones oculares a los 11 años después del nacimiento. En la radiografía se observan calcificaciones cerebrales de los plexos coroideos. En los casos graves es común encontrar al recién nacido con hidrocefalia y los signos y síntomas de encefalitis aguda, retinocoroiditis y anormalidades en el LCR. Las manifestaciones viscerales pueden existir pero no son predominantes. Más tarde se encuentran las manifestaciones intracraneales y se observa retardo psicomotor $(1,27)$.

En los casos en que la infección se produce al principio del embarazo, cuando se está formando la placenta, el parásito pasa al feto y se desarrolla la enfermedad en la vida intrauterina. Toda la infección generalizada y los daños ocurren en el feto y en el momento del nacimiento ya el niño tiene las secuelas. En las formas leves, las manifestaciones aparecen después del nacimiento, en la edad escolar y aún más tarde. En otros casos se encuentran lesiones más graves pero con manifestaciones tardías, como epilepsia, retardo en el desarrollo neurosíquico, retinocoroiditis y calcificaciones cerebrales. En los casos severos puede nacer el niño con macrocefalia, microcefalia, retraso en su desarrollo psicomotor, microftalmia, estrabismo, cataratas y glaucoma $(1,3$, 4, 26-28).

\section{RESPUESTA INMUNE Y DIAGNÓSTICO.}

Para interpretar correctamente los resultados de las pruebas serológicas debemos relacionarlo con la dinámica de la respuesta inmune ante este parásito. En la primoinfección se producen de inicio anticuerpos anti-T. gondii de las clases IgA e IgM, que son los marcadores que se relacionan con la fase aguda de la enfermedad. El tiempo de durabilidad en la detección de estas inmunoglobulinas en suero depende de la sensibilidad del método de laboratorio empleado. La IgG comienza a detectarse en bajas concentraciones cerca del mes del comienzo de la infección y su incremento es más lento que el de la $\operatorname{IgA}$ e $\operatorname{IgM}$, pero alcanza valores superiores hasta pasados los 6 meses, donde se mantiene un tiempo en meseta para después decaer hasta valores bajos (pero detectables por ELISA) que se mantendrán estables e indican infección pasada, inmunidad y protección para el feto $(18,29)$.

Idealmente, el tamizaje para la detección de anticuerpos de tipo IgG anti- Toxoplasma debe hacerse en el período preconcepcional. Esto permite detectar a las mujeres con títulos positivos de IgG específica y que, por lo tanto, ya han sufrido una primoinfección antes del embarazo quedando inmunizadas para el resto de sus vidas. Este grupo de pacientes no requerirá más estudios para toxoplasmosis al embarazarse, excepto en las pacientes que estén infectadas con el virus de inmunodeficiencia humana $(18,29)$.

Las gestantes seronegativas a Toxoplasma deberán checarse en cada trimestre del embarazo. En caso de que ocurra seroconversión se inicia tratamiento placentario y se solicita la prueba de reacción en cadena de la polimerasa (PCR) en líquido amniótico después de la semana 20 de gestación para descartar infección fetal $(30,31)$.

Cuando la paciente es IgG positiva en el embarazo y se desconoce IgG preconcepcional, se recomienda solicitar IgG dos semanas después y solicitar IgM preferiblemente en la misma muestra. Los resultados pueden ser:

a) Si los títulos de IgG permanecen estables con IgM negativa, se considera infección pasada. No se requiere tratamiento ni más controles.

b) Si los títulos de IgG se duplican y la IgM es positiva, se confirma infección reciente: se inicia tratamiento placentario y se solicita PCR en líquido amniótico.

c) Si los títulos de IgG se duplican y la IgM es negativa, se solicita IgA, nueva IgM (si está disponible) y puede realizarse el test de avidez para IgG. Un resultado de IgA negativo, no descarta la enfermedad y debe procederse a iniciar el tratamiento placentario

\section{Revista Biomédica}




\section{Toxoplasmosis congénita.}

y solicitar PCR en líquido amniótico.

Cuando la paciente es IgG negativa y la IgM es positiva, se debe repetir el examen a las tres semanas:

a) Si la IgG se torna positiva, se demuestra Toxoplasmosis reciente. En ciertos casos, la síntesis de IgG es evidente alrededor de una semana después de una prueba negativa. El tratamiento placentario y un diagnóstico prenatal están justificados en estos casos.

b) Si la IgG persiste negativa, se puede excluir la infección por Toxoplasma, excepto en pacientes inmunosuprimidas, caso en el cual también se justifica el tratamiento placentario y el diagnóstico prenatal.

La detección de $\operatorname{IgE}$ aún se mantiene poco estudiada. Esta inmunoglobulina parece ser prometedora como marcador de infección adquirida recientemente. Datos limitados sugieren que la detección de estos anticuerpos en adultos con infección aguda es breve en el tiempo, aun más que los niveles de IgM e IgA (29-40).

En general, la búsqueda serológica de anticuerpos IgG e IgM se realiza por ensayos como: inmunofluorescencia indirecta (IFI), prueba inmunoenzimática (ELISA), hemaglutinación indirecta (HAI) y la técnica de ISAGA. Desafortunadamente, la confiabilidad de las pruebas comerciales varía de forma considerable (41).

La seguridad en la detección de seroconversión en embarazadas es de gran importancia, primero porque un falso positivo puede ocasionar un aborto innecesario, en países en que tal práctica es permitida, y segundo porque la terapéutica inmediata evitaría cuadros graves para el feto. Es preciso tener seguridad en la identificación de los marcadores serológicos como la rápida elevación de los títulos de $\operatorname{IgM}$, IgA e $\mathrm{IgE}$, pues son estos los indicadores de la toxoplasmosis adquirida.

El diagnóstico prenatal de infección por $T$. gondii es recomendado cuando se establece un diagnóstico de toxoplasmosis adquirida en la gravidez o antes de la concepción y es muy sugestivo teniendo como referencia resultados de pruebas serológicas. Por lo tanto, han sido utilizadas técnicas que evidencien la presencia del parásito como: inoculación en ratones de sangre fetal obtenida por cordocentesis o de líquido amniótico tomado después de la decimoctava semana de gestación. Este es el método más sensible de aislamiento pero se precisan de 3 a 6 semanas para ser obtenidos los resultados. Desafortunadamente, esto lo convierte en poco práctico para el diagnóstico de rutina $(41,42)$.

Otra de las metodologías empleadas es el cultivo celular que, aunque requiere de menos tiempo ( 4 a 5 días), es menos sensible. Mayor sensibilidad (92\%) y especificidad que los métodos anteriores muestra la PCR. Esta técnica como el Dotblot parecen ser muy prometedoras, sobre todo porque brindan un diagnóstico preciso de la infección fetal antes de las 20 semanas de gestación. Esto es de vital importancia pues en la actualidad el diagnóstico serológico de infección fetal por $T$. gondii antes de las 20 semanas no es recomendado por razones técnicas, así como porque las respuestas de anticuerpos específicos (IgG e IgA) fetales sólo son detectables luego de la maduración del sistema inmune (41).

Otros hallazgos de laboratorio pueden sugerir infección fetal, como por ejemplo: aumento de IgM total, eosinofilia, plaquetopenia, elevación de la concentración de gamma-glutamiltransferasa y deshidrogenasa láctica. La ultrasonografía puede revelar anormalidades como la dilatación de ventrículos cerebrales, el engrosamiento de la placenta y hepatomegalia. La presencia de hidrocefalia y calcificaciones cerebrales es característica pero no es patognomónica de toxoplasmosis congénita $(25,41$, 43).

La enfermedad en el recién nacido puede ser inadvertida en el momento del nacimiento, pero se puede manifestar meses o incluso años después. En los casos subclínicos lo ideal sería la demostración de parasitemia en el neonato, lo que podría ser realizado mediante técnicas de cultivo celular, aislamiento de parásitos en animales o PCR de sangre venosa (4149).

El aislamiento del protozoario y la inmunohistoquímica del material de la placenta son también de gran valor para el diagnóstico. En relación con el diagnóstico serológico, la presencia de 


\section{Martín-Hernández.}

anticuerpos $\operatorname{IgM}$ en el neonato, puede ser de gran valor pues significa producción por él mismo. Por eso, si no es detectado al inicio, se debe repetir la prueba un mes después del nacimiento, porque la producción de IgM puede ser tardía. Para la realización de la técnica se deben eliminar los anticuerpos IgG, pues se pueden obtener resultados falsos negativos por acción competidora de estos, o falsos positivos por acción del factor reumatoideo, lo que es mucho más frecuente en neonatos (41-49).

La presencia de IgA también ha sido valorizada en el diagnóstico de la toxoplasmosis congénita. La presencia de IgG en el neonato, de hasta 10 días de nacido, debe ser evaluada con cuidado por causa de la IgG materna de transmisión pasiva. Por otro lado, la clínica sugestiva en el neonato, asociada con un cuadro serológico materno, es indicativa de infección reciente y tiene valor predictivo de infección congénita. En el suero del recién nacido la presencia de títulos elevados de anticuerpos $\operatorname{IgG}$, que aumentan o se negativizan, en un período de hasta 18 meses, es indicativo de toxoplasmosis congénita.

El diagnóstico postnatal incluye además continuo examen oftalmológico y neurológico, este último mediante tomografía axial computarizada (TAC) de cráneo (41-49).

\section{TRATAMIENTO Y PROFILAXIS.}

Una vez comprobada por medio de las diferentes técnicas la infección por Toxoplasma, se debe instaurar el tratamiento pleno, que logre modificar el curso de la enfermedad y disminuir las secuelas fetales. Los esquemas recomendados para este tipo de tratamiento se elegirán, según la disponibilidad en cada sitio de trabajo y tolerancia de los respectivos medicamentos.

Existen varios fármacos que han demostrado ser eficaces en el tratamiento de la toxoplasmosis congénita, entre los que se encuentran: pirimetamina, espiramicina y sulfadiazina (50-54).

La pirimetamina se administra por vía oral, penetra bien en el LCR y produce bloqueo secuencial en el metabolismo del ácido fólico o folínico por activación de inhibidores enzimáticos en diferentes puntos de la vía metabólica. Es depresor de la médula ósea y puede ocasionar trombocitopenia y a veces anemia y leucopenia, por lo que en el transcurso del tratamiento se debe controlar hemograma dos veces por semana. Para evitar su efecto tóxico, debe suministrarse ácido fólico por vía oral o intramuscular (50).

En la embarazada con infección aguda se recomienda pirimetamina después de las primeras semanas del embarazo y generalmente se realiza el tratamiento combinado con sulfadiazina. Las dosis más indicadas son: pirimetamina ( $1 \mathrm{mg} / \mathrm{kg} /$ día; máximo 75 $\mathrm{mg}$ /día, vía oral, en dos dosis diarias por 4 semanas) + sulfadiazina (120 mg/kg/día; máximo 4 g/día, vía oral, en cuatro dosis al día, por 4 semanas) + ácido fólico ( $5 \mathrm{mg} /$ día, vía oral, una dosis, por 4 semanas) $(51,52)$.

En el recién nacido con toxoplasmosis congénita se recomienda pirimetamina $1 \mathrm{mg} / \mathrm{kg} /$ día durante 3 días, seguido de $1 \mathrm{mg} / \mathrm{kg}$ cada 2 días + sulfadiazina $100 \mathrm{mg} / \mathrm{kg} /$ día en dos dosis + ácido fólico $5 \mathrm{mg}$. oral, dos veces por semana. La duración del tratamiento debe decidirse en cada caso por los especialistas; generalmente es de 6 meses a 1 año. Este régimen se puede administrar por 21 días, alternado con 4 semanas de espiramicina $100 \mathrm{mg} / \mathrm{kg} /$ día en tres dosis. Se recomienda el uso de corticoides sólo en casos de coriorretinitis progresiva que afecte la mácula (prednisona $1.5 \mathrm{mg} / \mathrm{kg} /$ día) $(49,50,53)$.

La espiramicina es una droga de toxicidad mínima y sin efectos teratógenos, por lo que es el fármaco de elección para el tratamiento de la toxoplasmosis en el embarazo. Es altamente efectiva contra el $T$. gondii y si bien sus niveles en sangre del cordón sólo alcanzan el $50 \%$ de los niveles séricos maternos, se concentra de manera importante en la placenta, alcanzando niveles de hasta cuatro veces los niveles séricos maternos. En embarazadas se recomiendan $3 \mathrm{~g}$ diarios divididos en 4 dosis por 4 semanas $(50,24)$.

El porcentaje de éxito terapéutico no es completo y entre $7 \%$ y $25 \%$ de los niños tratados in útero requieren tratamiento posterior, dependiendo si se usa terapia adicional con pirimetamina-sulfadiazina o espiramicina sola.

Las gestantes seronegativas a T. gondii deberán

\section{Revista Biomédica}




\section{Toxoplasmosis congénita.}

checarse cada trimestre del embarazo y extremar las medidas higiénicas-sanitarias como son:

-Cocción adecuada de los alimentos y en especial de las carnes.

-Beber agua potable.

-Lavarse las manos con agua y jabón antes de ingerir alimentos.

-Lavar las verduras y frutas antes de consumirlas.

-Cuando trabaje con tierra, protegerse con guantes y máscara.

-Tener especial cuidado con los gatos y en especial con sus heces fecales. Si tiene que realizar el cambio de su arena higiénica, debe realizarlo con máscara y guantes. Después lavarse bien las manos. Mantener los gatos dentro de la casa para que no salgan de cacería y alimentarlos con carnes bien cocidas $(50,54)$.

\section{CONCLUSIONES.}

La educación orientada a las mujeres en edad fértil y durante el embarazo, es una conducta que evita la adquisición de la infección. En países como Francia, Bélgica y Suecia se estima que la tasa de infección durante el embarazo está en descenso gracias al progreso en los informes sobre la higiene de los alimentos, pues casi todas las infecciones se deben a la costumbre de ingerir carne insuficientemente cocida (55).

El programa de descubrimiento de casos durante el embarazo tiene como principal, pero no único obstáculo, el costo de las pruebas serológicas a todas las embarazadas que acuden al control prenatal. Otros inconvenientes son el seguimiento y terapia de las madres con la infección reciente que exigen la existencia de un equipo multidisciplinario con técnicas actuales disponibles para demostrar la infección fetal, como la PCR. Inclusive, si se dispone de estas técnicas, es necesario el seguimiento cuidadoso y a largo plazo de los recién nacidos.

Aunque descubrir los casos en las madres y/o en los recién nacidos sea una obligación normal cuando existe el presupuesto y la infraestructura necesaria, la realidad del terreno induce a buscar alternativas como podrían ser: la realización de encuestas a las madres para descubrir a las que se encuentren expuestas a riesgo de contraer toxoplasmosis reciente, y el control de los gatos callejeros por parte de la salud pública. El éxito de un programa de control de la toxoplasmosis está estrechamente ligado al desarrollo del programa materno-infantil. Una primera actividad es la toma de conciencia por parte del cuerpo médico para realizar la prevención frente a la embarazada. En segundo lugar, extender la cobertura de las pruebas serológicas al mayor número posible de mujeres en gestación.

En algunos países como Francia y Austria, de acuerdo con lo que expresa la ley, el monitoreo serológico es obligatorio. Tal procedimiento ha reducido la incidencia de casos de toxoplasmosis fetal con seroconversión de 40 a $7 \%$. En otros como en Cuba y Brasil el examen de rutina no es realizado, la infección materna sólo se diagnostica cuando la indicación serológica es ejecutada por petición médica, conducta no generalizada; o por la sospecha clínica de infección aguda $(41,56)$.

En nuestro país se realizan esfuerzos por disminuir o erradicar las causas de mortalidad infantil, por lo que resulta importante considerar la aplicación de las pruebas de diagnóstico que permiten detectar en estados tempranos, en la madre o su descendencia la presencia o no de anticuerpos contra este agente infeccioso.

\section{REFERENCIAS.}

1.- Botero D, Restrepo M. Toxoplasmosis. En: Botero D, Restrepo M, editores. Parasitosis Humana. 2a ed. Medellín: Corporación para Investigaciones Biológicas; 1992. p 23148.

2.- Kasper LH. Toxoplasma infection. In: Braunwald E, Fauci AS, Kasper DL, Hauser SL, Longo DL, Jameson JL, editors. Harrinson's Principles of Internal Medicine. 15th edition. New York: McGraw-Hill 2001. p. 1222-7.

3.- Remington JS, McLeod R, Desmonts G. Toxoplasmosis. En: Remington JS, Klein JO, editores. Infectious diseases of the fetus and newborn. 5th ed. Philadelphia: WB Saunders; 2001. p 205-346.

4.- Wong SY, Remington JS. Toxoplasmosis in pregnancy. Clin Infect Dis 1994; 18: 853-62. 


\section{Martín-Hernández.}

5.- Valente P, Sever J. Infecciones congénitas diagnosticadas por muestreo fetal directo. Rev Hosp Niños B Aires 1993; 35: 187-97.

6.- Allain JP, Palmer CR, Pearson G. Epidemiological study of latent and recent infection by Toxoplasma gondii in pregnant women from a regional population in the U.K. J Infect 1998; 36: 189-96.

7.- Morten L, Larcen SO, Petersen E. Prevalence, incidence and geographical distribution of Toxoplasma antibodies in pregnant women in Denmark. Scand J Infect Dis 1993; 25: 751-6.

8.- Lappalainen M, Koskela P, Hedman K, Teramo K, Ammala $\mathrm{P}$, Hiilesmaa V, et al. Incidence of Primary Toxoplasma infections during pregnancy in Southern Finland: A prospective cohort study. Scand J Infect Dis 1992; 24: 97104.

9.- Lebech M, Larsen SO, Petersen E. Prevalence, incidence, and geografical distribution of Toxoplasma gondii antibodies in pregnant women in Denmark. Scand J Dis 1993; 25: 751-3.

10.- Jenum PA, Stray-Pedersen B, Melby KK, Kapperud G, Whitelaw A, Eskild A, et al. Incidence of Toxoplasma gondii infection in 35940 pregnant women in Norway and pregnancy outcome for infected women. J Clin Microbiol 1998; 36: 29006.

11.- Jenum PA, Kapperud G, Stray-Pedersen B, Melby KK, Eskild A, Eng J. Prevalence of Toxoplasma gondii specific immunoglobulin $\mathrm{G}$ antibodies among pregnant women in Norway. Epidemiol Infect 1998; 120: 87-92.

12.- Logar J, Novak-Antolic Z, Zore A, Cerar V, Likar M. Incidence of the congenital toxoplasmosis in the Republic of Slovenia. Scand J Infect Dis 1992; 24: 105-8.

13.- Amendoeira MRR, Souza WJS, Paulino AM, Bonifacio AC, Cordeiro JCA, Vicente RT, et al. Toxoplasmosis prevalence and potencial risk of congenital transmission in Rio de Janeiro - Preliminary results. Mem Inst Oswaldo Cruz 1995; 90(Supl 1): 290.

14.- Jones J, López A, Wilson M. Congenital toxoplasmosis. Am Fam Physician 2003; 67: 2131-8.

15.- Ambroise-Thomas P, Schweitzer M, Pinon JM, Thiebaugeorges O. Prevention of congenital toxoplasmosis in France. Risk assessment. Results and perspectives of prenatal screening and newborn follow up. Bull Acad Natl
Med 2001; 185: 665-83.

16.- Evengard B, Petersson K, Engman ML, Wiklund S, Ivarsson SA, Teariaknehjelm $\mathrm{K}$, et al. Low incidence of toxoplasma infection during pregnancy and in newborns in Sweden. Epidemiol Infect 2001; 127: 121-7.

17.-Mozzatto L, Procianoy RS. Incidence of congenital toxoplasmosis in Southern Brazil: a prospective study. Rev Inst Med Trop Sao Paulo 2003; 45: 147-51.

18.- González T, Molina JR. Toxoplasmosis congénita. Rev Cubana Obstet Ginecol 1997; 23: 7-12.

19.- López R., Pérez X, Guerra E, Herrera R, Acosta C. Toxoplasmosis entre mujeres embarazadas en Ciudad de la Habana. Biomed 1993; 13: 173-8.

20.- González T, Bacallao J, García C, Molina JR. Prevalencia de anticuerpos anti- Toxoplasma gondii en una población de mujeres embarazadas en Cuba. Gac Méd Méx 1991; 131 : 499-503.

21.- Acosta C, Pérez X, García R. Presencia de anticuerpos IgG anti- Toxoplasma gondii en embarazadas residentes en Ciudad de la Habana. Rev Biomed 2001; 12: 250-4.

22.- Sánchez A, Martín I, García SM. Estudio de reactividad a Toxoplasma gondii en embarazadas de las provincias Ciudad de la Habana y Pinar del Río, Cuba. Bioquimia 2003; 111:3-8.

23.- Petersen E, Pollak A, Reiter-Owona I. Recent trends in research on congenital toxoplasmosis. Int J Parasitol 2001; 31:115-44.

24.- Vergani P. Congenital toxoplasmosis: efficacy of maternal treatment with Spiramycin alone. Am J Reprodu Inmmunol 1998; 39: 335-40.

25.- Daffos F, Forestier F, Capella-Pavlovsky M, Thulliez P, Aufrant C, Valenti D, et al. Prenatal management of 746 pregnancies at risk for congenital toxoplasmosis. N Engl J Med 1988; 318: 271-5.

26.- Crino JP. Ultrasound and fetal diagnosis of perinatal infection. Clin Obstet Gynecol 1999; 42: 71-80.

27.- Machín R,. Bravo JR, Mitjans A, Cordoví R. Toxoplasmosis II. Algunos aspectos relativos a trastornos oculares. Rev. Cub. Med. Trop 1984; 12: 305-14.

28.- Gónzalez I, Díaz M, Pérez J. Coriorretinitis por 


\section{Toxoplasmosis congénita.}

Toxoplasma en niños. Rev Cubana Med Trop 1999; 51:13842

29.- Jenum PA, Stray-Pedersen B . Development of specific immunoglobulins G, M and a following primary Toxoplasma gondii infection in pregnant women. J Clin Microbiol 1998; 36: 2907-13.

30.- Jenum PA, Holberg-Petersen M, Melby KK, StrayPedersen B. Diagnosis of congenital Toxoplasma gondii infection by polymerase chain reaction (PCR) on amniotic fluid samples. APMIS 1998; 106: 680-6.

31.- Grover CM, Thulliez P, Remington JS, Boothroyd JC. Rapid prenatal diagnosis of congenital Toxoplasma infection by using polymerase chain reaction and amniotic fluid. J Clin Microbiol 1990; 28:297-301.

32.- Jenum PA, Stray-Pedersen B, Gudersen A. Improved diagnosis of primary Toxoplasma gondii infection in early pregnancy by determination of anti-Toxoplasma immunoglobulin G avidity. J Clin Microbiol 1997; 35: 1972-7.

33.- Lappalainen M, Koskela P, Koskiniemi M, Ämmälä P, Hiilesmaa V, Teramo K, et al. Toxoplasmosis acquired during pregnancy improved serodiagnosis based on avidity of IgG. J Infect Dis 1993; 167: 691-7.

34.- Cozon GJ, Ferrandiz J, Nebhi H, Wallon M, Peyron F. Estimation of the avidity of inmunuglobulin $\mathrm{G}$ for routine diagnosis of chronic Toxoplasma gondii infection in pregnant women. Eur J Clin Microbiol Infec Dis 1998; 17: $32-6$.

35.- Foudrinier F, Marx-Chemla C, Aubert D, Bonhomme A, Pinon JM. Value of specific immunoglobulin A detection by two immunocapture assay in the diagnosis of toxoplasmosis. Eur J Clin Microbiol Infect Dis 1995; 14: 585-90.

36.- Arcavi M, Orfus G, Griemberg G. Diagnosis of toxoplasmosis by joint of inmunoglobulin $\mathrm{A}$ and inmmunoglobulin M. J Clin Microbiol 1997; 35: 1450-3.

37.- Ashburn D, Joss AW, Pennington TH, Ho Yen D. Do $\operatorname{IgA}, \operatorname{IgE}$, and $\operatorname{IgG}$ avidity test, have any value in the diagnosis of Toxoplasma infection in pregnancy? J Clin Pathol 1998; 51: 312-5.

38.- Gross U, Henkel O, Darde ML. Value of detecting immunoglobulin $\mathrm{E}$ antibodies for the serological diagnosis of Toxoplasma gondii infection. Clin Diagn Lab Inmunol 1997; 4: 247-51.
39.- Sobieszczanka B., Rdzka A. Detection of IgA antibodies as important markers of acute primary infection with Toxoplasma gondii. Pol Merkuriusz Lek 1997 ; 3: 228-30.

40.- Hohlfeld P, Biedermann K, Extermann P, Gyr T. Toxoplasmosis in pregnancy: Prevention, prenatal diagnosis and treatment. Schweiz Med Wochenschr Suppl 1995; 65: $62-9$.

41.- Reis MR. Diagnóstico de la toxoplasmosis congénita. Rev Cubana Invest Biomed 2001; 20: 118-21.

42.- Fricker A, Pelloux H, Muet F, Racinet C, Bost M, GoullierFleuret A, et al. Prenatal diagnosis of congenital toxoplasmosis: comparative value of fetal blood and amniotic fluid using serological techniques and cultures. Prenat Diag 1997; 17: 831-5.

43.- Camargo ME. Alguns aspectos actuais do diagnóstico de laboratório da toxoplasmose. An Acad Nac Med 1995; 155: 236-9.

44.- Holliman RE. Congenital toxoplasmosis: prevention, screening, and treatment. J Hosp Infect 1995; 30: 179-90.

45.- Bader TJ, Macones GA, Aschi DA. Prenatal screening for toxoplasmosis. Obstet Gynecol 1997; 90: 457-64.

46.- Eaton RB. Evaluation of neonatal Toxoplasma gondii IgM FEIA. J Clin Microbiol 1996; 34: 3147-50.

47.- Pinon JM, Chemla C, Villena I, Foudrinier F, Aubert D, Puygauthier-Toubas D, et al. Early neonatal diagnosis of congenital toxoplasmosis: value of comparative enzymelinked immunofiltration assay immunological profiles and anti- Toxoplasma gondii immunoglobulin M (IgM) or IgA immunocapture and implications for postnatal therapeutic strategies. J Clin Microbiol 1996; 34: 579-83.

48.- Newton ER. Diagnosis of perinatal TORCH infections. Clin Obstet Gynecol 1999;42, 59-70.

49.- Guerina NG, Hsu H, Meissner HC, Maguire JH, Lynfield $\mathrm{R}$, Stechenberg B, et al. Neonatal screening and early treatment for congenital Toxoplasma gondii infection. N Engl J Med 1994; 330: 1858-963.

50.- Valdés MC, Díaz AG, Svarch N. Actualidades en el tratamiento y profilaxis de la toxoplasmosis. Rev Cubana Med Gen Inegr 1996; 12: 4-6.

51.- Matsui D. Prevention, diagnosis, and treatment of fetal toxoplasmosis. Clin Perinatol 1994; 21: 675-89.

Vol.15/No.3/Julio-Septiembre, 2004 


\section{Martín-Hernández.}

52.- Foulon W, Villena I, Stray P, Decoster A., Lappalainen M., Pinon JM, et al. Treatment of toxoplasmosis during pregnancy: a multicenter study of impact on fetal transmision and children's sequelate at 1 year. Am J Obstet Gynec 1999; 180 (2 Pt 1): 410-5.

53.- Villena I. Pyrimethamine-Sulfadoxine treatment of congenital toxoplasmosis:follow-up of 78 cases between 1980 and 1997. Reims Toxoplasmosis Group. Scand J Infec Dis 1998; 30: 295-300.

54.- Kapperud G, Jenum PA, Stray- Pedersen B, Melby KK, Eskild A, Eng J. Risk factors for Toxoplasma gondii infection in pregnancy. Am J Epidemiol 1996; 144: 405-12.

55.- Desmonts G. Congenital Toxoplasmosis: A prospective study of 378 pregnancies. N Engl J Med 1974; 290: 1110.

56.- Hohlfeld P, Daffos F, Thulliez P, Aufrant C, Couvreur J, McAleese Descombey D, et al. Fetal toxoplasmosis: outcome of pregnancy and infant follow-up after in utero treatment. J Pediatr 1989; 115: 765-9. 